\title{
«Es dürfte sich lohnen, den Patienten vermehrt zuzuhören»
}

\author{
Das eben erschienene Buch «Mit einer Erbkrankheit leben» versteht sich als Rat- \\ geber, der Betroffenen und deren Angehörigen, aber auch Fachleuten Unterstüt- \\ zung bieten und wichtige Informationen vermitteln möchte. Mitherausgeber \\ Hansjakob Müller, emeritierter Ordinarius für medizinische Genetik an der Univer- \\ sität Basel, gibt im Interview Auskunft zu den Anliegen und Absichten des Werks.
}

Interview: Bruno Kesseli

${ }^{*}$ VHL $=$ Von-Hippel-LindauErkrankung

bkesseli[at]emh.ch

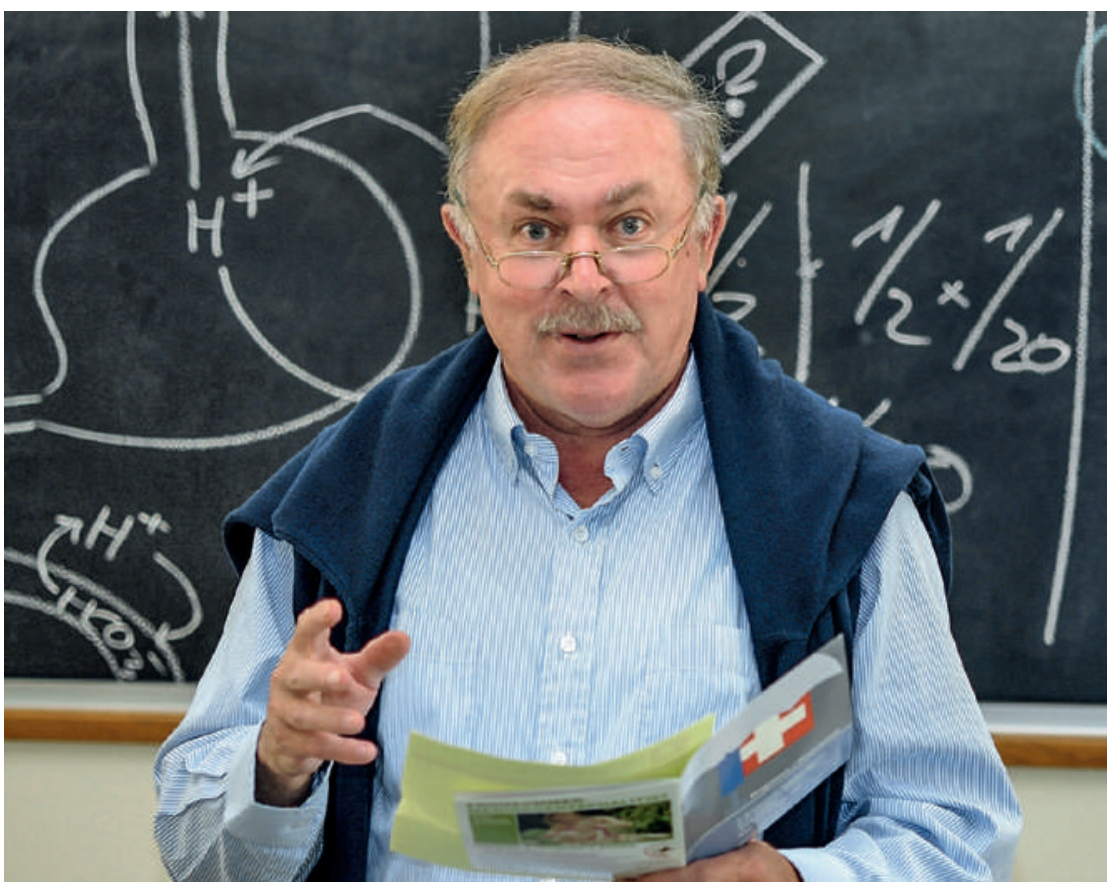

Hansjakob Müller: Für Patienten mit Erbkrankheiten ist es nachteilig, dass in der Schweiz die Medizinische Genetik bei der Aus-, Weiter- und Fortbildung recht stiefmütterlich behandelt wird.
Das Buch enthält einerseits Beiträge von Fachvertretern der Medizin und der Genetik, der Psychologie, des Rechts und der Ethik, der Familienplanung und der Sozialversicherungen, andererseits Erfahrungsberichte von Personen mit Erbkrankheiten. Wie äussern sich diese über ihre Ärztinnen und Ärzte?

Der Umgang mit Personen, die eine krankheitsverursachende, durchschlagskräftige Veranlagung haben, ist zweifelsohne für die Ärzteschaft nicht immer einfach. In den Erfahrungsberichten werden jene Ärztinnen und Ärzte besonders gelobt, die auf die Ratsuchenden eingehen und sich ernsthaft um ihre Sorgen kümmern. Auch die gute zwischenmenschliche Atmosphäre im Paraplegikerzentrum Nottwil fand mehrfach Anerkennung. Aber eben, es kam auch anderes zutage!

Was sprechen Sie konkret an?

Beim Aufzeichnen der Gespräche wurde offensichtlich, was Patienten alles mitbekommen, gerade dann, wenn sie wegen ihrer Krankheit regelmässig eine Praxis oder ein Spital aufsuchen müssen. Dort machen sie nicht nur ihre eigenen Beobachtungen, sondern tauschen diese mit anderen Betroffenen aus. Es dürfte sich lohnen, den Patienten vermehrt zuzuhören, um diese Informationsquelle für Verbesserungen unseres Medizinalwesens zu nutzen.

Welche Kritiken sind für die Ärzteschaft von besonderem Interesse?

Die einzelne Erbkrankheit ist selten. So verfügt man vielerorts kaum über eigene Erfahrungen im Umgang mit einer solchen «Orphan disease», erinnert sich vielleicht noch aus dem Medizinstudium an deren Namen. Zudem besteht oft eine geringe Bereitschaft, sich darüber in der nicht unmittelbar greifbaren Fachliteratur oder über geeignete Internetquellen zu informieren und sich mit den individuellen Sorgen der Ratsuchenden auseinanderzusetzen. Es wirkt sich offensichtlich nachteilig aus, dass in der Schweiz die Medizinische Genetik bei der Aus-, Weiter- und 
Fortbildung recht stiefmütterlich behandelt wird. Dabei betonen alle Betroffenen, wie wichtig es für sie ist, von Fachleuten betreut zu werden, die sich über das Wesen ihrer Erbkrankheit, aber auch das Leben mit einer solchen auskennen.

\section{«Analog zu den vielerorts gut eingespielten «Tumor- boards` sind an grösseren Spitälern «Genetikboards〉 einzurichten.»}

Wie sollen Praktiker mit dieser Situation umgehen? Fehlen solche Kenntnisse, sollte man die Patienten an eine Kollegin / einen Kollegen respektive an ein medizinisches Zentrum überweisen, wo diese eher vorhanden sind. Gelegentlich muss man als Arzt einfach über den eigenen Schatten springen, wenn man feststellt, dass die/der Ratsuchende über einen bestimmten Sachverhalt oder über die praktische Durchführung einer Untersuchung mehr weiss. Es ist zu bedenken, dass Patienten sich beachtliches medizinisches Wissen, ob am eigenen Leib erfahren oder bei Angehörigen beobachtet, aneignen und Erfah-

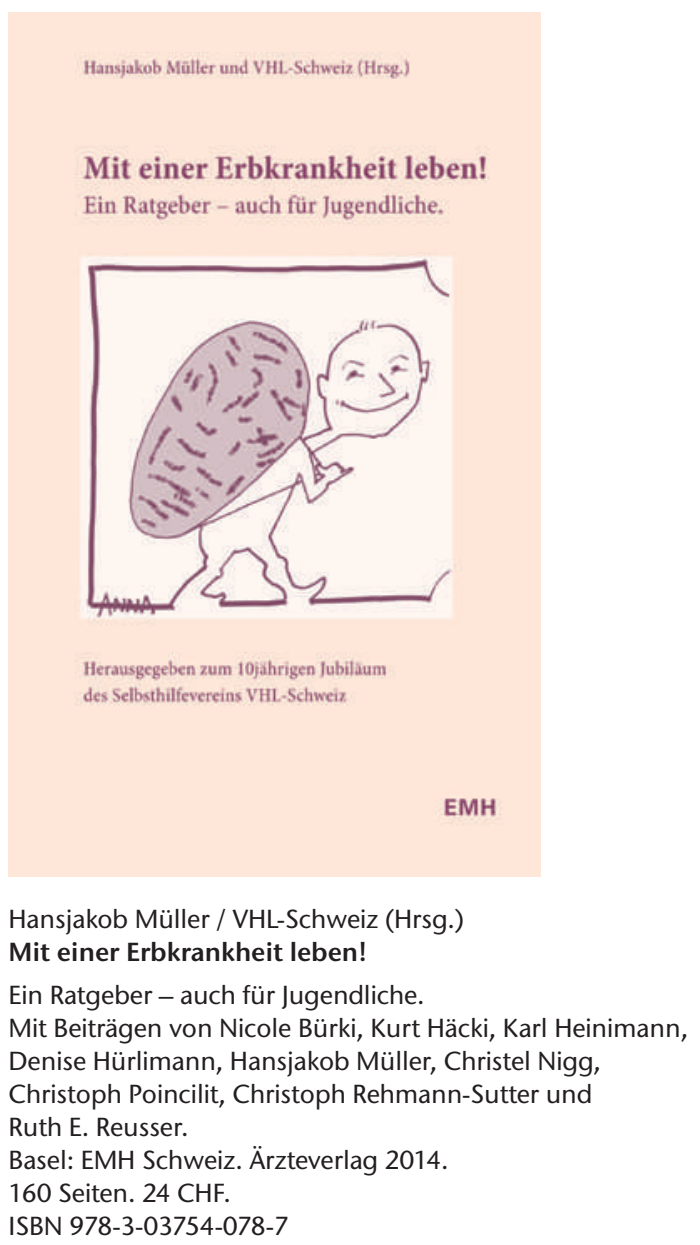

rungen in der Vorgehensweise bei medizinischen Massnahmen sammeln. Zudem informieren sie sich über ihre Krankheit im Internet respektive im Schosse von Selbsthilfeorganisationen. Patienten, gerade auch junge, wollen mit ihrem eigenen Wissen ernst genommen werden.

Können Sie einige Punkte nennen, die für Betroffene im Umgang mit Ärzten und medizinischen Institutionen besonders schwierig sind?

Bemühend ist es für die Betroffenen, wenn sie bei den regelmässigen Kontrolluntersuchungen einem unerfahrenen Assistenzarzt immer wieder alles von neuem erzählen müssen oder in den verschiedenen Wartezimmern eines medizinischen Zentrums viel Zeit ungenutzt verstreicht und wenn sie nicht klar wissen, wer dort ihr eigentlicher Ansprechpartner ist. Für sie wäre ein kompetenter «Lotsen-Dienst» sehr hilfreich, der die Aktivitäten der verschiedenen Fachspezialisten koordiniert und die notwendigen Kontakte schafft, gerade dann, wenn mehrere Organe überwacht werden müssen.

Wie könnte man die oft komplexe ärztliche Betreuung von Personen mit Erbkrankheiten verbessern?

Analog zu den vielerorts gut eingespielten «Tumorboards» sind an grösseren Spitälern «Genetikboards» einzurichten, wo man sich über Befunde und angezeigte medizinische Massnahmen beim einzelnen Patienten orientieren und dann gemeinsam das weitere Vorgehen koordinieren kann. Es ist wünschenswert, dass die an Universitäts- und Kantonsspitälern für die umfassende Betreuung von Patienten mit einer bestimmten Erbkrankheit benötigten Fachleute sich zu einem eigentlichen Zirkel zusammentun, um sich dort nicht nur regelmässig über indizierte Kontrolluntersuchungen oder Therapien austauschen, sondern auch, um sich gegenseitig fortbilden zu können. So lässt sich frustrierender Leerlauf für alle, Patienten und Betreuer, reduzieren.

\section{Sehen Sie weitere Schwachstellen der Betreuung?}

Gelegentlich sind die zugezogenen Spezialisten über die bereits bestehenden Krankenakten schlecht informiert. Eine Ärztin wollte bei der Augenhintergrundskontrolle beharrlich auch das linke Auge einer jungen Frau mit der Von-Hippel-Lindau-Erkrankung untersuchen. Sie hatte vorgängig nicht mitbekommen, dass dieses wegen Hämangioblastomen durch ein Glasauge ersetzt werden musste. Auch die fehlende Erfahrung von Spezialärzten und deren Teams im Umgang mit Kindern kann zu Problemen führen. Nicht selten wird der ungenügende Informationsfluss zwischen den Spezialisten im Zentrum und den Hausärzten bemängelt. Letztere erhalten Berichte mit «nackten» medizinischen Fakten und erfahren nicht, was alles mit den Patienten diskutiert wurde und welche Massnahmen aus welchen Gründen man ihnen vorschlug. 
Die medizinische Information ist das eine - wie sieht es mit der psychosozialen Betreuung aus?

Immer wieder wird berichtet, dass die medizinischen und genetischen Informationen über eine Erbkrankheit von ärztlicher Seite gut waren, dass man dann aber die Betroffenen nach deren Vermittlung allein liess und diese sich mit all ihren Nöten und Sorgen selber weiterhelfen mussten. So erging es einem Patienten mit dem Marfan-Syndrom, der vom Arzt beim Abschluss des Gespräches erfuhr, dass er bald einmal erblinden werde. Auf sein Befinden nach dieser Mitteilung nahm er keine Rücksicht. Eine Ehefrau suchte gemeinsam mit ihrem an der Huntington-Erkrankung leidenden Mann wegen zunehmenden Eheschwierigkeiten eine psychiatrische Sprechstunde auf. Im Verlauf des Gespräches rastete der Patient aus und verliess wutentbrannt den Raum. Man empfahl der Frau darauf, sich scheiden zu lassen. Ein Rat, wie sie sich in der aktuellen Konfliktsituation verhalten soll, blieb aus. Man entliess sie ohne entsprechende Hilfe.

Fühlen sich die Spezialisten allenfalls für diesen Bereich nicht zuständig?

Die Betroffenen bemerkten mehrfach, dass häufig eine eigentliche Kluft zwischen der Ärzteschaft und dem Pflegepersonal besteht und dass man sich gegenseitig wenig über die gesundheitlichen Probleme, aber auch die Befindlichkeit der Patienten austauscht. Mancherorts wird die «seelische» Betreuung einfach der Pflege überlassen.

\section{«Mancherorts wird \\ die «seelische> Betreuung einfach der Pflege überlassen.»}

Wenn wir von der Kritik weg zu einem positiven Ansatz kommen möchten: Worauf ist bei der Betreuung von Patienten mit einer Erbkrankheit zu achten?

Ein guter Arzt ist vorerst zweifelsohne die medizinische Fachperson, dann aber auch Fürsorger, Ratgeber, Vermittler, gelegentlich Mahner, z.B. dann, wenn wichtige Vorsorgemassnahmen wieder einmal versäumt wurden. Gerade männliche Anlageträger tun sich mit dem Einhalten regelmässiger Kontrolluntersuchungen oft schwer! Die Vermittlung medizinischgenetischer Fakten, vor allem dann, wenn sie mit unangenehmen Aspekten verbunden sind, erfordert viel Zeit, Geduld, Einfühlungsvermögen, Taktgefühl und letztlich auch Ehrlichkeit. Betroffene klammern sich leicht an falsche Hoffnungen. Dann muss man sie sachte von solchen und den damit verbundenen Erwartungen abbringen. Auch ärztliche Notfalldienste müssen auf die Möglichkeiten und Bedürfnisse von Behinderten Rücksicht nehmen können, die nicht in der Lage sind, eigenständig eine Notfallstation aufzusuchen oder telefonischen Anweisungen zu folgen.

Zum Schluss: Gibt es einen allgemeinen Rat, den Sie Patienten mit einer Erbkrankheit geben möchten?

Patienten mit Erbkrankheiten, ob blind, taub oder im Rollstuhl, sollten angeregt werden, die Öffentlichkeit nicht zu meiden. Nur durch den regelmässigen Kontakt mit ihnen lässt sich das Verständnis für behinderte Mitmenschen in unserer Bevölkerung fördern. Eine Person mit Gangschwierigkeiten wegen einer Muskelkrankheit ist nicht gleich ein Betrunkener. So ist keineswegs nur die Ärzteschaft im Hinblick auf einen besseren Umgang mit Behinderten herausgefordert, erwähnt seien auch zahlreiche weitere Berufsgruppen wie die Verantwortlichen für den Bus- und Eisenbahnverkehr oder die Besitzer von Hotels und Restaurants! Darüber erfährt man im Ratgeber mehr.

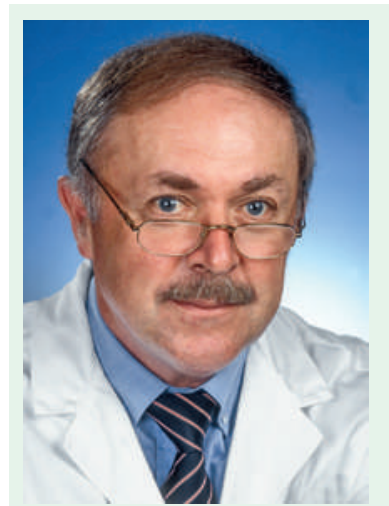

\section{Hansjakob Müller}

Hansjakob Müller ist Professor emeritus für Medizinische Genetik an der Universität Basel. Er war Leiter der Abt. Medizinische Genetik des Universitätskinderspitals beider Basel (UKBB) und des Dept. Biomedizin USB. Seine Forschungsinteressen galten vor allem Veranlagungen für Tumorkrankheiten. Er war Mitglied von nationalen und internationalen Ethikkommissionen. Als ärztlicher Beirat unterstützt er Selbsthilfeorganisationen für Erbkrankheiten. Die Information der Öffentlichkeit über den Fortschritt der Biomedizin, speziell der Medizinischen Genetik, sowie die breite Diskussion der damit verbundenen wissenschaftlichen, ethischen und psychosozialen Fragen sind ihm ein besonderes Anliegen.

\section{VHL Schweiz}

Der VHL-Schweiz ist der Verein für von der Von-Hippel-Lindau (VHL)-Erkrankung betroffene Familien in der Schweiz. 